\title{
Laser Wakefield Driven Generation of Isolated Carrier-Envelope-Phase Tunable Intense Subcycle Pulses
}

\author{
E. Siminos $\odot,^{1, *}$ I. Thiele, ${ }^{2, \dagger}$ and C. Olofsson ${ }^{1,2}$ \\ ${ }^{1}$ Department of Physics, University of Gothenburg, SE-412 96 Göteborg, Sweden \\ ${ }^{2}$ Department of Physics, Chalmers University of Technology, SE-412 96 Göteborg, Sweden
}

(Received 22 March 2020; revised 2 September 2020; accepted 4 January 2021; published 29 January 2021)

\begin{abstract}
Sources of intense, ultrashort electromagnetic pulses enable applications such as attosecond pulse generation, control of electron motion in solids, and the observation of reaction dynamics at the electronic level. For such applications, both high intensity and carrier-envelope-phase (CEP) tunability are beneficial, yet hard to obtain with current methods. In this Letter, we present a new scheme for generation of isolated CEP tunable intense subcycle pulses with central frequencies that range from the midinfrared to the ultraviolet. It utilizes an intense laser pulse that drives a wake in a plasma, copropagating with a longwavelength seed pulse. The moving electron density spike of the wake amplifies the seed and forms a subcycle pulse. Controlling the CEP of the seed pulse or the delay between driver and seed leads to CEP tunability, while frequency tunability can be achieved by adjusting the laser and plasma parameters. Our 2D and 3D particle-in-cell simulations predict laser-to-subcycle-pulse conversion efficiencies up to $1 \%$, resulting in relativistically intense subcycle pulses.
\end{abstract}

DOI: 10.1103/PhysRevLett.126.044801

Electromagnetic pulses containing less than a single oscillation of the electromagnetic field are unique tools for the investigation and exploitation of nonadiabatic phenomena. One of the most prominent examples is the generation of attosecond pulses [1], the efficiency of which depends on the carrier-envelope-phase (CEP) of the driver pulse [2]. Moreover, an intense driver is particularly advantageous since it can produce even shorter (e.g., zeptosecond [3]) pulses. Isolated subcycle pulses with both CEP tunability and high energy are very attractive, not only for attosecond pulse generation, but also many other applications in solid-state physics [4,5] and nanoengineering [6]. Therefore, developing methods to obtain isolated CEP tunable high-intensity subcycle pulses is an active field of recent research. At present, solid-state lasers delivering relativistic intensity pulses are limited to durations above one and a half cycle [7], while scaling the intensity of subcycle pulses produced by parametric amplification methods remains challenging [8], especially in the mid-IR [9].

Plasma-based methods, which are scalable even to relativistic intensities, offer hope to resolve this issue. In the context of attosecond pulse generation in the XUV regime, several techniques have been developed in order to

Published by the American Physical Society under the terms of the Creative Commons Attribution 4.0 International license. Further distribution of this work must maintain attribution to the author(s) and the published article's title, journal citation, and DOI. Funded by Bibsam. produce isolated pulses, such as polarization [10-12] or intensity gating [13-16] and exploitation of wave front rotation [17,18]. More recently, plasma-based methods have been proposed to generate longer wavelength, e.g., mid-IR, single and subcycle pulses. We have shown that electron beams can be used to generate intense subcycle pulses, by amplifying a seed pulse reflected by a foil [19]. Another technique to generate midinfrared, near-singlecycle pulses, which exploits the laser frequency downconversion known to appear in laser driven wakefields, has been proposed in [20]. However, all these plasma-based techniques are either not CEP tunable or require a controllable CEP-stable high-intensity laser, which is technically challenging.

In this Letter, we demonstrate that isolated CEP tunable intense subcycle pulses can be created by a frequency upconversion process, which we refer to as laser wakefield driven amplification (LWDA). We propose to inject a CEPstable long-wavelength seed pulse of relatively low intensity in copropagation with a high-intensity, not necessarily CEP-stable, driver laser pulse into a gas jet. The driver pulse ionizes the gas already at its rising edge and creates a plasma. Because of the ponderomotive force, the highintensity laser pulse (driver) displaces the plasma electrons, creating a charge separation field, which leads to plasma oscillations behind the laser pulse. These wakefield oscillations [21,22] are strongly anharmonic for sufficiently intense drivers and form electron density spikes after each oscillation period. In the highly nonlinear regime, the first period is completely void of electrons (forming the socalled bubble) and the first electron density spike is most 


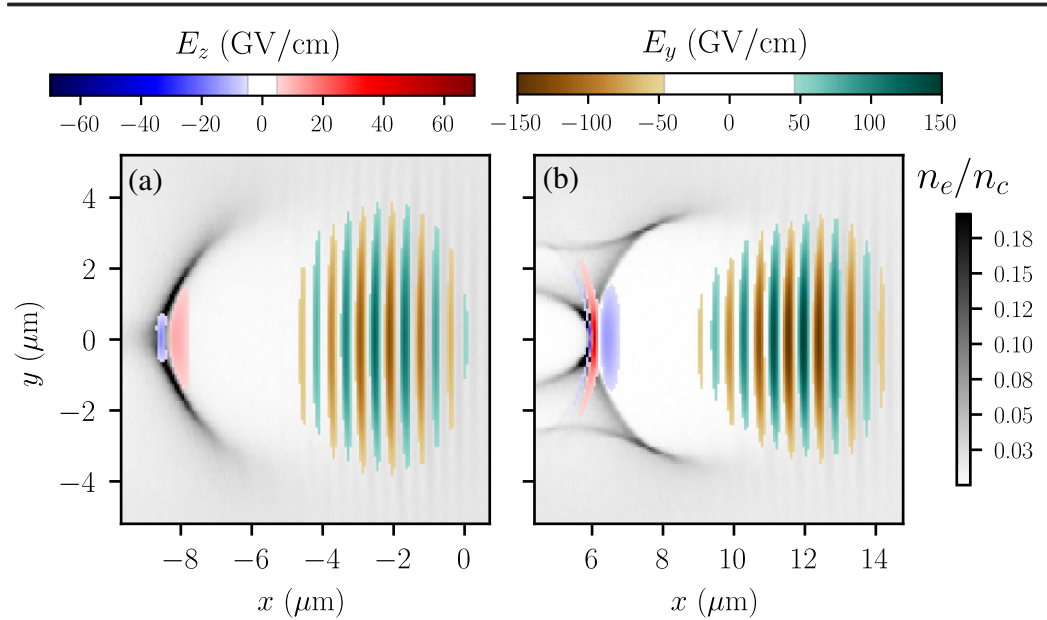

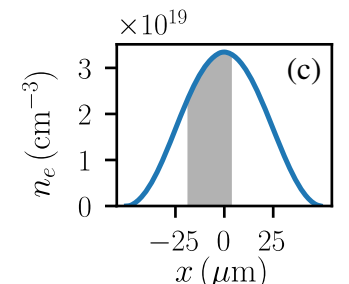
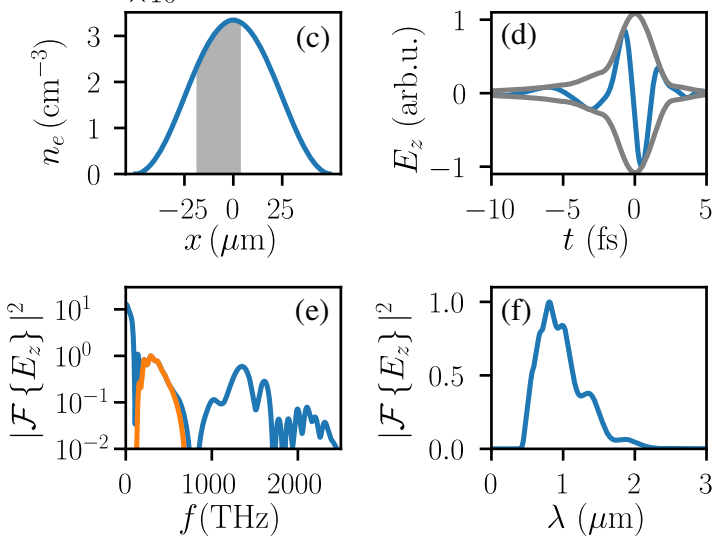

FIG. 1. Results of a 3D particle-in-cell simulation demonstrating LWDA. Cross sections of the plasma density and electromagnetic field in the driver $\left(E_{y}\right)$ and seed $\left(E_{z}\right)$ polarization direction, (a) before and (b) after wave breaking. (c) Density profile used in the simulations, with the gray-shaded area showing the region where amplification occurs. (d) On-axis electric field of the subcycle pulse at the exit of the gas jet. (e) Power spectrum of the amplified pulse at the exit of the gas jet, with the part of the spectrum retained after filtering shown in orange. (f) The retained power spectra after filtering as a function of wavelength.

pronounced. In the scheme proposed here, it is this electron density spike, moving close to the speed of light, that amplifies the seed pulse, leading to intense subcycle-pulse generation.

We demonstrate subcycle-pulse generation by means of a 3D particle-in-cell (PIC) simulation (see Fig. 1) using the code SMILEI [23]. A $y$-polarized driving laser pulse and a $z$-polarized seed pulse are injected along the $x$ direction. They are defined by their transverse electric field components $E_{y}$ and $E_{z}$, respectively, according to

$$
E_{y / z}\left(\mathbf{r}_{\perp}, t\right)=E_{0, L / S} e^{-\frac{y^{2}+z^{2}}{r_{0, L / S}^{2}}} \sin \left[\phi_{L / S}(t)\right] f_{L / S}(t),
$$

with amplitudes $E_{0, L / S}$, beam waists $r_{0, L / S}$, angular frequencies $\omega_{0, L / S}$, phases $\phi_{L / S}(t)=\omega_{0, L / S} t+\phi_{0, L / S}$, with variable seed CEP $\phi_{0, S}$, driver CEP $\phi_{0, L}=0$, and envelope of the form

$$
f(t)=\exp \left(-2 \ln (2) t^{2} / t_{0, L / S}^{2}\right)
$$

The simulation parameters are summarized in Table I. The gas jet is modeled as a preionized plasma with immobile ions and a cosine-squared electron density profile, see Fig. 1 , with peak density $n_{0}=0.019 n_{c}=3.3 \times 10^{19} \mathrm{~cm}^{-3}$, where $n_{c}=\epsilon_{0} m_{e} \omega_{0, L}^{2} / q_{e}^{2}$ is the critical density and $q_{e}$ and $m_{e}$ are the electron charge and mass, respectively. The gas jet has a short length scale with diameter (FWHM) $L_{p}=50 \mu \mathrm{m}$, which is experimentally feasible [24] (longer gas jets can be used, as will be shown). The driver laser has relativistic strength, characterized by normalized peak vector potential $a_{0, L}=q_{e} E_{0, L} / m_{e} c \omega_{0, L}=2.5$ (intensity $I_{0, L}=1.3 \times 10^{19} \mathrm{~W} / \mathrm{cm}^{2}$ ). Its duration $t_{0, L}=11 \mathrm{fs}$ satisfies $c t_{0, L}<\lambda_{p e}$, where $\lambda_{p e}=2 \pi c / \omega_{p e}$ is the electron plasma wavelength and $\omega_{p e}=\sqrt{n_{0} q_{e}^{2} / m_{e} \epsilon_{0}}$ is the plasma frequency. This ensures that a wake with a pronounced density spike in the first period is excited [22]. The seed pulse wavelength has been chosen to correspond to $\lambda_{0, S}=0.7 \lambda_{p e}=4 \mu \mathrm{m}$ in order to ensure propagation of the seed, $\omega_{0, S}>\omega_{p e}$, while at the same time guaranteeing that the seed wavelength is longer than the electron skin depth, $\lambda_{0, S}>c / \omega_{p e}=\lambda_{p e} / 2 \pi$, which can be taken as an upper bound for the density spike characteristic length scale [25]. The seed is subrelativistic $\left(a_{0, S}=0.1\right)$, while its energy is $\mathcal{E}_{0, S}=0.3 \mathrm{~mJ}$, which is well within reach of optical parametric amplification [26].

Figure 1(a) shows how the first electron density spike of the wake behind the laser pulse amplifies the seed pulse and forms a subcycle pulse. The second and subsequent electron density spikes also create subcycle pulses. However, these have an intensity at least one order of magnitude smaller than that of the leading pulse, which is amplified by the dominant electron density spike, resulting in an isolated subcycle pulse. Amplification occurs predominantly over a distance of approximately $20 \mu \mathrm{m}$ in the rising edge of the gas jet [gray-shaded area in Fig. 1(c)], where the high-density gradient significantly enhances the leading density spike, while suppressing wave breaking [27]. Amplification is interrupted shortly after the seed enters the declining part of the jet, due to wave breaking associated with the plasma wavelength increase in the downramp [28], see Fig. 1(b). The peak electric field of the seed is amplified by a factor of 84 from 0.8 to $67 \mathrm{GV} / \mathrm{cm}$, while its peak wavelength is downshifted to $0.8 \mu \mathrm{m}$ [see Fig. 1(f)]. With respect to this wavelength, the peak field of the subcycle pulse is relativistic, $a_{0 \text {,sub }} \simeq 1$.7. The subcycle pulse is subsequently guided within the elongated plasma bubble until it exits the plasma, [Fig. 1(d)] with an 
TABLE I. Parameters used in the simulations for the plasma (see text), driver, and seed laser parameters in Eq. (1) (and also the corresponding pulse energies $\mathcal{E}_{0, L / S}$ ) and discretization parameters: longitudinal $N_{x}$ and transverse $N_{y}=N_{z}$ resolution in number of cells per (driver) wavelength, temporal resolution $N_{t}$ in number of steps per optical cycle, and number of particles per cell $N_{p}$.

\begin{tabular}{|c|c|c|c|c|c|c|c|c|c|c|c|c|c|c|c|c|}
\hline $\operatorname{Fig}(\mathrm{s})$. & $n_{0}\left(\mathrm{~cm}^{-3}\right)$ & $L_{p}(\mu \mathrm{m})$ & $\lambda_{p e}(\mu \mathrm{m})$ & $\mathcal{E}_{0, L}$ & $\mathrm{a}_{0, L}$ & $t_{0, L}(\mathrm{fs})$ & $r_{0, L}(\mu \mathrm{m})$ & $\lambda_{0, S}(\mu \mathrm{m})$ & $\mathcal{E}_{0, S}$ & $a_{0, S}$ & $t_{0, S}$ & $r_{0, S}(\mu \mathrm{m})$ & $N_{x}$ & $N_{y}$ & $N_{t}$ & $N_{p}$ \\
\hline 1 & $3.3 \times 10^{19}$ & 50 & 5.8 & $60 \mathrm{~mJ}$ & 2.5 & 11 & 4.8 & 4 & $0.3 \mathrm{~mJ}$ & 0.1 & $78 \mathrm{fs}$ & 27 & 35 & 15 & 50 & 5 \\
\hline 2 & $4.5 \times 10^{19}$ & $\ldots$ & 5 & $39 \mathrm{~mJ}$ & 2.5 & 10 & $\ldots$ & 4 & $\ldots$ & 0.005 & $64 \mathrm{fs}$ & $\ldots$ & 105 & $\ldots$ & 120 & $\ldots$ \\
\hline $3(\mathrm{a})$ & $4.5 \times 10^{19}$ & 23 & 5 & $39 \mathrm{~mJ}$ & 2.5 & 10 & 4.2 & 4 & $\ldots$ & 0.005 & $\mathrm{cw}$ & 5 & 50 & 25 & 57 & 100 \\
\hline $4(\mathrm{~b}), 4(\mathrm{~d})$ & $1.75 \times 10^{18}$ & 152 & 25 & $5 \mathrm{~J}$ & 2.5 & 50 & 21 & 20 & $\ldots$ & 0.005 & $\mathrm{cw}$ & 25 & 50 & 25 & 57 & 100 \\
\hline
\end{tabular}

ultrabroad spectrum, intensity FWHM duration of $\sim 2$ fs or 0.75 cycles, and polarization orthogonal to the driver. The latter property allows the subcycle and driver pulses to be separated after the interaction. Most of the spectral power of the subcycle pulse lies within $\sim 2$ octaves from 0.45 to $2 \mu \mathrm{m}$ [Fig. 1(f)]. Note that spectral components outside the regime of interest for this subcycle pulse have been filtered out, see Fig. 1(e) and the Supplemental Material [29]. The electromagnetic energy in the polarization plane of the seed pulse increases by $0.2 \mathrm{~mJ}$ at the exit of the plasma $(0.1 \mathrm{~mJ}$ lies within the spectral range of the subcycle pulse).

In order to describe the amplification process in simpler terms, we develop a 1D model based on relativistic cold fluid theory. The propagation of the seed in the plasma can be described by the wave equation

$$
\partial_{x x}^{2} A_{z}-\partial_{t t}^{2} A_{z}=n_{e} A_{z} / \gamma_{e}=n_{0} A_{z} /(1+\phi),
$$

where $\phi, n_{e}$, and $\gamma_{e}$ are the wake scalar potential, electron fluid density, and relativistic factor, respectively; $A_{z}$ is the seed vector potential and $n_{0}$ is the background plasma density, in relativistic units [29]. In the last step, use has been made of the fact that in the quasistatic approximation for the driver laser propagation $n_{e} / \gamma_{e}=n_{0} /(1+\phi)$ [30]. The scalar potential for the nonlinear wake is determined by numerically solving $[22,30,31]$

$$
\frac{d^{2} \phi}{d \xi^{2}}=n_{0}\left(\frac{1+A_{y}^{2}}{2(1+\phi)^{2}}-\frac{1}{2}\right)
$$

where $A_{y}$ is the vector potential of the driver laser, $\xi=x-v_{g} t$, and $v_{g} \simeq c\left(1-1.5 n_{0} / n_{c}\right)$ is the driver group velocity [32]. The first period of the steady-state wakefield solution propagating at $v_{g}$ is shown in Fig. 2(a). The solution of Eq. (4) for $\phi$ is used in order to solve Eq. (3) numerically. As shown in Fig. 2(d), the model predicts rapid seed wavelength decrease, as well as significant electromagnetic field energy gain. Figure 2(b) shows that the wavelength downshift and energy gain is associated with localized amplification at the front of the density spike of the wake. This is consistent with the prediction of Ref. [19], that a pulse can gain energy from the declining part (with respect to $x$ ) of a subwavelength, moving density perturbation. This effect is distinct from the frequency upshift or "photon acceleration" [33-37], associated with electromagnetic field propagation in a plasma with (spatially) decreasing density. In the latter case, the pulse wavelength is shorter than the density variation length scale and pulse energy increase is only possible through an associated pulse length increase [34]. Nevertheless, also in our case plasma propagation effects are important and a purely beam driven description [19] does not apply. Moreover, the amplification process is not due to a parametric process such as stimulated Raman scattering [38], since the driver and seed polarization are orthogonal. Finally, note that LWDA is distinct from the formation of optical bullets in laser wakefield acceleration, which occurs when a short-wavelength probe is trapped inside the plasma density depression (bubble) created by the driver laser $[39,40]$.
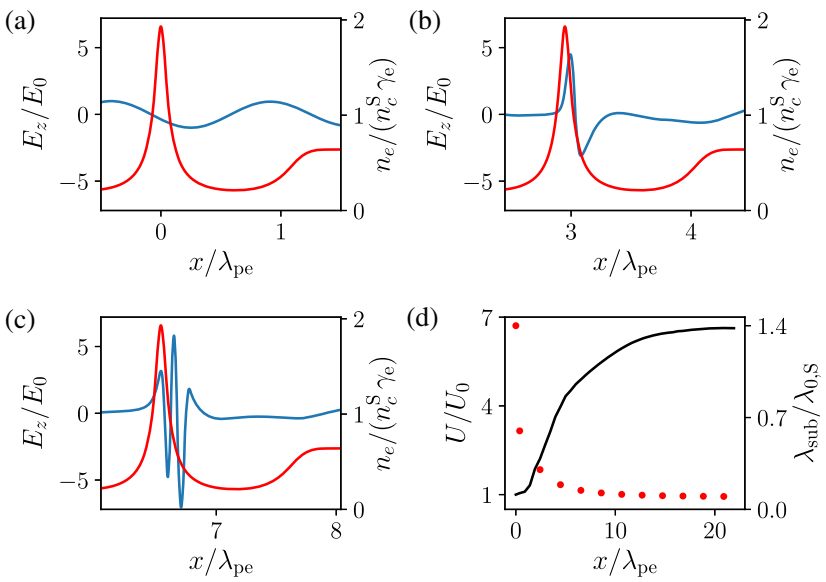

FIG. 2. Amplification in the fluid model. (a)-(c) Seed electric field $E_{z}$, normalized to the initial seed amplitude $E_{0}$ (blue lines) and $n_{e} / \gamma_{e}=n_{0} /(1+\phi)$ from Eq. (4), normalized to the critical density $n_{c}^{S}$ of the long-wavelength seed (red lines) at different propagation distance. (d) Electromagnetic energy $U$ in the polarization plane of the seed (obtained by spatial integration of the corresponding energy density), normalized to the initial seed energy $U_{0}$ (black, solid line) and peak wavelength $\lambda_{\text {sub }}$ of the subcycle pulse, normalized to the seed vacuum wavelength $\lambda_{0, S}$ (red dots), as a function of propagation distance. For $x=0$, the wavelength corresponds to the seed peak wavelength in a homogeneous plasma. The parameters are given in Table I. 
After propagation for $\sim 5 \lambda_{p e}$, the rate of energy increase starts to decrease, since the local seed wavelength is not anymore longer than the density modulation length scale. Moreover, the increased group velocity, due to the decrease in wavelength, of the amplified seed implies that the latter starts to dephase with respect to the density spike, see Fig. 2(c), leading to a gradual pulse lengthening. In the 3D PIC simulations of Fig. 1, wave breaking occurs before dephasing becomes an issue and the subcycle pulse is guided within the bubble until the gas jet exit, allowing the use of gas jets of reasonable dimensions. We note that, while the static wakefield model captures very well the salient features of subcycle-pulse formation, amplification is higher in PIC simulations, due to the additional enhancement of the first density spike induced by the driver laser evolution in that case.

We also perform a parametric study with 2D simulations in a simplified setting in order to reduce computational costs, while still capturing the main effects (see Supplemental Material [29] for a comparison of 2D and 3D results). We consider a flattop plasma density profile, the seed pulse is modeled within the continuous wave approximation $(\mathrm{cw})$, $f_{S}(t)=1$ in Eq. (1), and the remaining simulation parameters are summarized in Table I. Figure 3(a) shows the generated subcycle pulse at the exit of the plasma with halfcycle duration and peak wavelength of $\lambda_{\text {sub }}=0.4 \mu \mathrm{m}$.

In order to tune the CEP of the subcycle pulse, it is sufficient to introduce a delay $t_{d}$ of the many-cycle seed pulse with respect to the laser pulse or to change the CEP of the seed $\phi_{S}$. In the cw approximation studied here, these two operations are equivalent, since $t_{d} \omega_{0, S}=\phi_{0, S}$; the finite pulse duration case is studied in the Supplemental Material [29]. Figure 3(a) presents the on-axis electric field shapes after the interaction using four different seed pulse delays in steps of $\lambda_{0, S} / 8$ (or phase shifts of $\pi / 4$ ). It can be seen that the subcycle-pulse envelopes are the same, however, the phases of the subcycle pulses are shifted by $\pi / 4$. The synchronization level necessary to achieve a change of $\pi / 2$ in CEP is $\lambda_{0, S} / 4$. For the range of densities studied here, this corresponds to $3-17 \mathrm{fs}$, which is within present experimental capabilities [41]. Moreover, we show in [29] that the subcycle phase is not sensitive to variations in the driver laser pulse duration or energy by up to $20 \%$, making such control strategy viable.
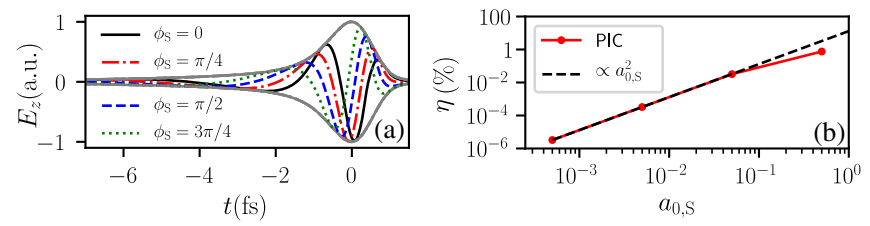

FIG. 3. Results of 2D PIC simulations. (a) On-axis electric fields of the amplified subcycle pulses after the interaction for different seed delays $t_{d}$ and their common envelope (gray line). (b) Energy conversion efficiency dependence on the peak seed electric normalized potential.
According to the cold fluid model (3), the interaction is linear in the seed transverse vector potential, implying a quadratic scaling of the energy conversion efficiency with the seed electric field amplitude. This is confirmed by our 2D PIC simulation results in Fig. 3(b), up to weakly relativistic seed pulse amplitudes. The suboptimal scaling for $a_{0, S}>0.1$ is caused by the feedback of the seed pulse on the wake. In this case, the undepleted driver approximation, implicit in Eq. (4), does not hold; the wake loses a substantial fraction of its energy to the seed pulse and $\phi$ in Eq. (3) cannot be considered to be stationary and independent of the subcycle field $A_{z}$ anymore. Note that, as discussed in relation to Fig. 1, relativistic field strengths can nevertheless be reached using subrelativistic seed pulses. Figure 3(b) shows that the laser-to-subcycle-pulse conversion efficiency $\eta$ reaches about $1 \%$.

The 1D model suggests that, apart from the interaction length, the subcycle pulses' spectra could be tuned with the driver-laser field strength $a_{0, L}$ and electron density $n_{0}$. The larger $a_{0, L}$ and $n_{0}$ are, the narrower the amplifying electron density spikes, which naturally leads to shorter wavelength components in the subcycle-pulse spectra, see Fig. 4. Tuning through the use of $a_{0, L}$ is limited by early wave breaking of the amplifying electron density spike above $a_{0, L}=3.5$ (for this particular plasma density). Tuning through the variation of $n_{0}$ is, according to our simulations, effective as long as $0.7 \lambda_{p e} \lesssim \lambda_{0, S} \lesssim \lambda_{p e}$.

In order to scale our results to a wider range of densities, one can fix the driver laser wavelength to $\lambda_{L}=0.8 \mu \mathrm{m}$ and
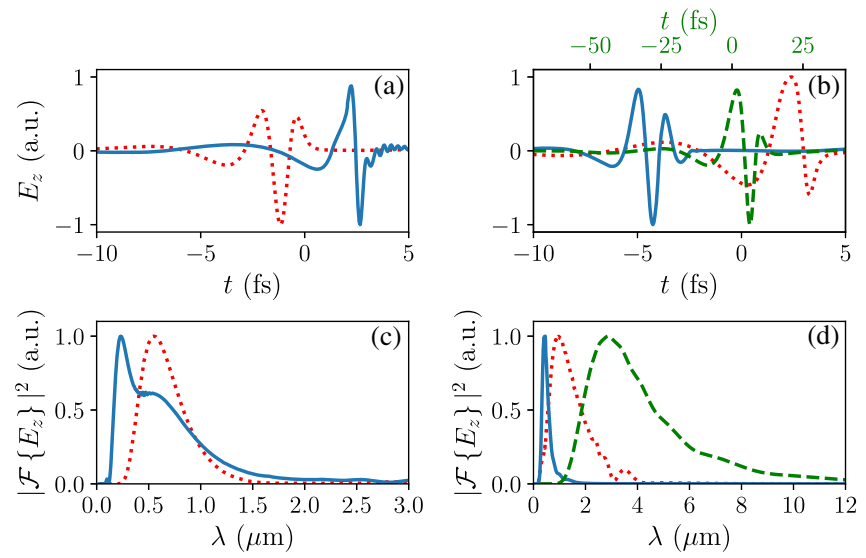

FIG. 4. Demonstration of the spectral tunability by modifying (a),(c) laser peak normalized vector potential from $a_{0, L}=2$ (red dotted lines) to $a_{0, L}=3.5$ (blue solid lines) and (b),(d) electron density from $n_{0}=0.019 n_{c}=3.35 \times 10^{19} \mathrm{~cm}^{-3}$ (red dotted lines) to $n_{0}=0.045 n_{c}=7.8 \times 10^{19} \mathrm{~cm}^{-3}$ (blue solid lines), keeping the other parameters the same as in Fig. 3. For the green dashed lines in (b), (d), the density has been reduced to $n_{0}=0.001 n_{c}=1.75 \times 10^{18} \mathrm{~cm}^{-3}$, while the rest of the parameters have been rescaled in proportion to $\lambda_{p e}$ (see Table I). Note the different timescale in this case. The figures present the (a),(b) on-axis electric fields of the subcycle pulses after the interaction, as well as (c),(d) their power spectra. 
vary $n_{0} / n_{c}$, while scaling all other parameters in proportion to the plasma wavelength. An example for low-density plasma, $n_{0}=0.001 n_{c}=1.7 \times 10^{18} \mathrm{~cm}^{-3}$, is shown as a green dashed line in Figs. 4(b) and 4(d), see Table I. Because of the longer seed wavelength used in this case, $\lambda_{0, S}=20 \mu \mathrm{m}$, the generated subcycle pulse lies in the midinfrared.

In summary, we propose a scheme for the generation of isolated, CEP tunable relativistic subcycle pulses by laser wakefield driven amplification of a seed electromagnetic pulse. The scheme has been shown to work over a wide range of plasma densities, utilizing seed pulses with wavelengths ranging from 4 to $20 \mu \mathrm{m}$ to produce relativistic subcycle pulses with peak wavelengths adjustable from the midinfrared to the ultraviolet.

The authors thank J. Ferri, M. Grech, L. Gremillet, G. Golovin, D. Guénot, V. Horný, M. Kaluza, and L. Veisz for helpful suggestions, T. Blackburn for a careful reading of the manuscript, and T. Fülöp for support. This work was supported by the Knut and Alice Wallenberg Foundation and by the Swedish Research Council, Grant No. 201605012. Numerical simulations were performed using computing resources at Grand Équipement National pour le Calcul Intensif (GENCI, Grants No. A0030506129 and No. A0040507594) and Chalmers Centre for Computational Science and Engineering (C3SE) provided by the Swedish National Infrastructure for Computing (SNIC, Grants No. 2017/1-484, No. 2017/1-393, No. 2018/3-297, No. 2018/2-13, and No. 2019/4-96).

*evangelos.siminos@physics.gu.se †illia-thiele@web.de

[1] F. Krausz and M. I. Stockman, Nat. Photonics 8, 205 (2014).

[2] P. Corkum and F. Krausz, Nat. Phys. 3, 381 (2007).

[3] C. Hernández-García, J. A. Pérez-Hernández, T. Popmintchev, M. M. Murnane, H. C. Kapteyn, A. Jaron-Becker, A. Becker, and L. Plaja, Phys. Rev. Lett. 111, 033002 (2013).

[4] G. Günter, A. A. Anappara, J. Hees, A. Sell, G. Biasiol, L. Sorba, S. De Liberato, C. Ciuti, A. Tredicucci, A. Leitenstorfer, and R. Huber, Nature (London) 458, 178 (2009).

[5] M. Hohenleutner, F. Langer, O. Schubert, M. Knorr, U. Huttner, S. W. Koch, M. Kira, and R. Huber, Nature (London) 523, 572 (2015).

[6] T. Rybka, M. Ludwig, M. F. Schmalz, V. Knittel, D. Brida, and A. Leitenstorfer, Nat. Photonics 10, 667 (2016).

[7] D. E. Rivas et al., Sci. Rep. 7, 5224 (2017).

[8] C. Manzoni, O. D. Mücke, G. Cirmi, S. Fang, J. Moses, S. Huang, K. Hong, G. Cerullo, and F. X. Kärtner, Laser Photonics Rev. 9, 129 (2015).

[9] H. Liang, P. Krogen, Z. Wang, H. Park, T. Kroh, K. Zawilski, P. Schunemann, J. Moses, L. F. DiMauro, F. X. Kärtner, and K.-H. Hong, Nat. Commun. 8, 141 (2017).

[10] T. Baeva, S. Gordienko, and A. Pukhov, Phys. Rev. E 74, 065401(R) (2006).
[11] P. Tzallas, E. Skantzakis, C. Kalpouzos, E. P. Benis, G. D. Tsakiris, and D. Charalambidis, Nat. Phys. 3, 846 (2007).

[12] M. Yeung, J. Bierbach, E. Eckner, S. Rykovanov, S. Kuschel, A. Sävert, M. Förster, C. Rödel, G. G. Paulus, S. Cousens, M. Coughlan, B. Dromey, and M. Zepf, Phys. Rev. Lett. 115, 193903 (2015).

[13] G. D. Tsakiris, K. Eidmann, J. Meyer-ter Vehn, and F. Krausz, New J. Phys. 8, 19 (2006).

[14] P. Heissler, R. Hörlein, J. M. Mikhailova, L. Waldecker, P. Tzallas, A. Buck, K. Schmid, C. M. S. Sears, F. Krausz, L. Veisz, M. Zepf, and G. D. Tsakiris, Phys. Rev. Lett. 108, 235003 (2012).

[15] G. Ma, W. Dallari, A. Borot, F. Krausz, W. Yu, G. D. Tsakiris, and L. Veisz, Phys. Plasmas 22, 033105 (2015).

[16] D. Kormin, A. Borot, G. Ma, W. Dallari, B. Bergues, M. Aladi, I. B. Fldes, and L. Veisz, Nat. Commun. 9, 4992 (2018).

[17] H. Vincenti and F. Quéré, Phys. Rev. Lett. 108, 113904 (2012).

[18] J. A. Wheeler, A. Borot, S. Monchoc, H. Vincenti, A. Ricci, A. Malvache, R. Lopez-Martens, and F. Qur, Nat. Photonics 6, 829 (2012).

[19] I. Thiele, E. Siminos, and T. Fülöp, Phys. Rev. Lett. 122, 104803 (2019).

[20] Z. Nie, C.-H. Pai, J. Hua, C. Zhang, Y. Wu, Y. Wan, F. Li, J. Zhang, Z. Cheng, Q. Su, S. Liu, Y. Ma, X. Ning, Y. He, W. Lu, H.-H. Chu, J. Wang, W. B. Mori, and C. Joshi, Nat. Photonics 12, 489 (2018).

[21] P. Gibbon, Short Pulse Laser Interactions with Matter: An Introduction (Imperial College Press, London, 2005).

[22] E. Esarey, C. B. Schroeder, and W. P. Leemans, Rev. Mod. Phys. 81, 1229 (2009).

[23] J. Derouillat, A. Beck, F. Pérez, T. Vinci, M. Chiaramello, A. Grassi, M. Flé, G. Bouchard, I. Plotnikov, N. Aunai, J. Dargent, C. Riconda, and M. Grech, Comput. Phys. Commun. 222, 351 (2018).

[24] J. Faure, D. Gustas, D. Guénot, A. Vernier, F. Böhle, M. Ouillé, S. Haessler, R. Lopez-Martens, and A. Lifschitz, Plasma Phys. Controlled Fusion 61, 014012 (2018).

[25] J. Thomas, I. Y. Kostyukov, J. Pronold, A. Golovanov, and A. Pukhov, Phys. Plasmas 23, 053108 (2016).

[26] D. Woodbury, L. Feder, V. Shumakova, C. Gollner, R. Schwartz, B. Miao, F. Salehi, A. Korolov, A. Pugžlys, A. Baltuška, and H. M. Milchberg, Opt. Lett. 43, 1131 (2018).

[27] J. Mu, F.-Y. Li, M. Zeng, M. Chen, Z.-M. Sheng, and J. Zhang, Appl. Phys. Lett. 103, 261114 (2013).

[28] C. G. R. Geddes, K. Nakamura, G. R. Plateau, C. Toth, E. Cormier-Michel, E. Esarey, C. B. Schroeder, J. R. Cary, and W. P. Leemans, Phys. Rev. Lett. 100, 215004 (2008).

[29] See Supplemental Material at http://link.aps.org/ supplemental/10.1103/PhysRevLett.126.044801 for review of the analytical model, comparison of 2D and 3D simulations, description of spectral filtering and additional simulations.

[30] P. Sprangle, E. Esarey, and A. Ting, Phys. Rev. A 41, 4463 (1990).

[31] S. V. Bulanov, V. I. Kirsanov, and A. S. Sakharov, JETP Lett. 50, 198 (1989). 
[32] W. Lu, M. Tzoufras, C. Joshi, F. S. Tsung, W. B. Mori, J. Vieira, R. A. Fonseca, and L. O. Silva, Phys. Rev. ST Accel. Beams 10, 061301 (2007).

[33] S. C. Wilks, J. M. Dawson, W. B. Mori, T. Katsouleas, and M. E. Jones, Phys. Rev. Lett. 62, 2600 (1989).

[34] E. Esarey, A. Ting, and P. Sprangle, Phys. Rev. A 42, 3526 (1990).

[35] L. Oliveira e Silva and J. T. Mendonça, Phys. Rev. E 57, 3423 (1998).

[36] C. D. Murphy et al., Phys. Plasmas 13, 033108 (2006).

[37] Z. Bu, B. Shen, L. Yi, H. Zhang, S. Huang, and S. Li, Phys. Plasmas 22, 043102 (2015).
[38] W. Kruer, The Physics of Laser Plasma Interactions (CRC Press, Boca Raton, 2003).

[39] Z.-M. Sheng, Y. Sentoku, K. Mima, and K. Nishihara, Phys. Rev. E 62, 7258 (2000).

[40] P. Dong, S. A. Reed, S. A. Yi, S. Kalmykov, G. Shvets, M. C. Downer, N. H. Matlis, W. P. Leemans, C. McGuffey, S. S. Bulanov, V. Chvykov, G. Kalintchenko, K. Krushelnick, A. Maksimchuk, T. Matsuoka, A. G. R. Thomas, and V. Yanovsky, Phys. Rev. Lett. 104, 134801 (2010).

[41] G. Golovin, W. Yan, J. Luo, C. Fruhling, D. Haden, B. Zhao, C. Liu, M. Chen, S. Chen, P. Zhang, S. Banerjee, and D. Umstadter, Phys. Rev. Lett. 121, 104801 (2018). 